How the latencies of excitation and inhibition determine ganglion cell thresholds and discharge patterns in the frog

Donner, K.

Elsevier

1981

Vision Research. 1981. 21: 1689-1692

http://hdl.handle.net/1975/928

http://dx.doi.org/doi:10.1016/0042-6989(81)90059-6

Downloaded from Helda, University of Helsinki institutional repository.

This is an electronic reprint of the original article.

This reprint may differ from the original in pagination and typographic detail.

Please cite the original version. 


\title{
HOW THE LATENCIES OF EXCITATION AND INHIBITION DETERMINE GANGLION CELL THRESHOLDS AND DISCHARGE PATTERNS IN THE FROG
}

\author{
KRISTIAN DONNER \\ Department of Zoology, Division of Physiology, University of Helsinki, \\ 00100 Helsinki 10, Finland
}

(Received 1 August 1980)

\section{INTRODUCTION}

The effects of inhibition on the responses of retinal ganglion cells are different in different states of adaptation. As was first observed in the cat by Barlow et al. (1957) and later in the frog by Donner and Reuter (1965), the spike threshold is more strongly raised by light-adaptation when measured with large stimuli extending into the inhibitory surround of the receptive field (RF) than with smaller stimuli. The experiments to be described were intended to decide whether, in the frog, this difference actually reflects a disappearance or decisive densitization of the inhibitory mechanism. It is found that threshold adaptation as well as discharge patterns may be accounted for largely in terms of the relative timing of excitatory and inhibitory signals without invoking any dramatic changes in the RF.

\section{RESULTS}

Thresholds and patterns of impulse discharges of ganglion cell on responses to $513 \mathrm{~nm}$ circular step stimuli of different sizes and intensities were registered extracellularly, in the dark-adapted state and at various times after full-field bleaches. Figure 1 shows that threshold adaptation in the frog follows a similar pattern as in the cat: area-threshold curves from the dark-adapted eye usually fail to show any inhibitory influence from the surround, while in the lightadapted eye the thresholds obtained with large stimuli are significantly higher than those to somewhat smaller stimuli (Fig. 1a). And if the thresholds to a small and a large stimulus are followed through the course of dark-adaptation (Fig. 1b), the latter are seen to be relatively elevated in the beginning, but drop rather abruptly at some time during adaptation (unrelated to the cone-rod shift). Thus there is an apparent disappearance of the inhibitory surround.

The presence of inhibition in the dark-adapted RF: discharge patterns

It is quite clear, however, that inhibition does play an important role even in the dark-adapted RF. This is seen from the full spike discharges to a series of test intensities elicited with stimulus spots of different diameters. Such recordings from a typical class 3 cell are displayed in Fig. 2 for four stimuli, one smaller than the excitatory centre (a), one roughly equal to it (b), one extending moderately beyond it (c) and a large one strongly stimulating the surround (d). The high-intensity responses to all test spots consist of an initial burst more or less sharply interrupted and followed by stray spikes only. It seems justified to think that the interruption is due to inhibition, as it is most prominent for the largest stimuli. It should be noted that for smaller stimuli the burst gets longer towards the lower intensities, while for large ones it gets shorter. For these, there is in fact no initial burst, only a few stray spikes at low intensities. These responses are obviously inhibited already close to the absolute threshold. Counting the total number of spikes in the responses further confirms this (Donner, 1981).

The constancy of discharge patterns during dark-adaptation

What, then, does actually change, when the thresholds to large stimuli make the transition from the light-adapted to the dark-adapted situation? In Fig. 3 is shown an experiment performed on a sustained cell, where the full discharges to a whole series of intensities were repeatedly registered during the course of adaptation. Threshold adaptation curves were measured in parallel. Two points emerge: (1) the high-intensity responses give no suggestion of a sudden "release from inhibition" as the threshold drop occurs, and (2) the decisive change is the appearance of a distinct low-intensity range of responses different in type from the short and fast high-intensity ones. The two ranges may remain separated by a silent intensity range, as in Fig. 3, persisting even in the dark-adapted state. Considering Figs $2 \mathrm{~d}$ and 3 , it seems that the long and irregular type of response to a large stimulus is possible only at low absolute stimulus intensities. Thus these responses start appearing only when the receptors have dark-adapted to a sufficient sensitivity. So the above observations give rise to two new questions: (1) why do these responses 


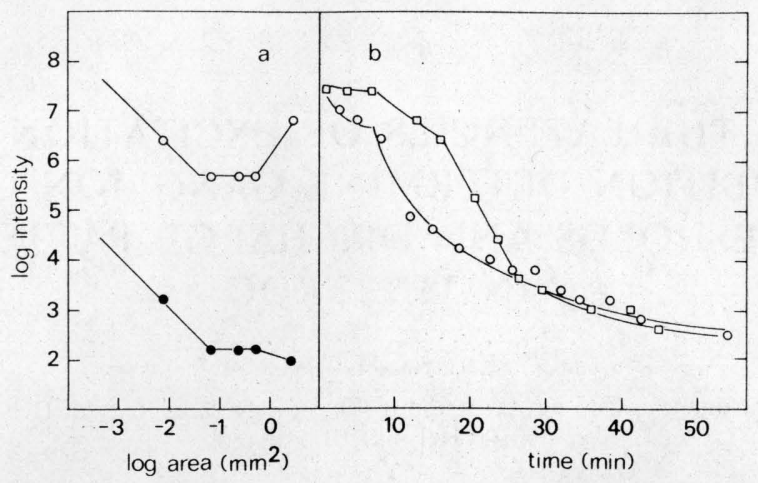

Fig. 1. (a) Threshold intensity as a function of the area of the stimulating spot for a class 3 unit in the dark-adapted state (lower) and $25 \mathrm{~min}$ after a $3 \%$ rhodopsin bleach (upper). (b) Threshold adaptation curves measured after a $1.5 \%$ bleach with test spots of $1.9 \mathrm{~mm}$ (squares) and $0.3 \mathrm{~mm}$ (circles) dia. Ordinate log threshold intensity (here and subsequently log intensity 10 means $8.4 \cdot 10^{9}$ quanta $/\left(\mathrm{sec} \cdot \mathrm{mm}^{2}\right)$ incident on the retina). Abscissa: time after bleach; Temperature: $10-12 \mathrm{C}$.

appear at the lowest intensities, in spite of inhibition, and (2) how are the elevated thresholds during the initial phase of adaptation determined? The first question will be only briefly touched upon in the Discussion (see Donner, 1981); instead here will be proposed a detailed answer to the second question. For this purpose it will be necessary to take a look at the temporal behaviour of the signals.

\section{The light-dark-adaptation of the latency to the first} spike

Since inhibition, in order to affect thresholds, must be not only strong enough, but also appropriately timed, one is led to consider the relative timing of the excitatory and inhibitory signals and its importance for response-formation in general. The latency to the

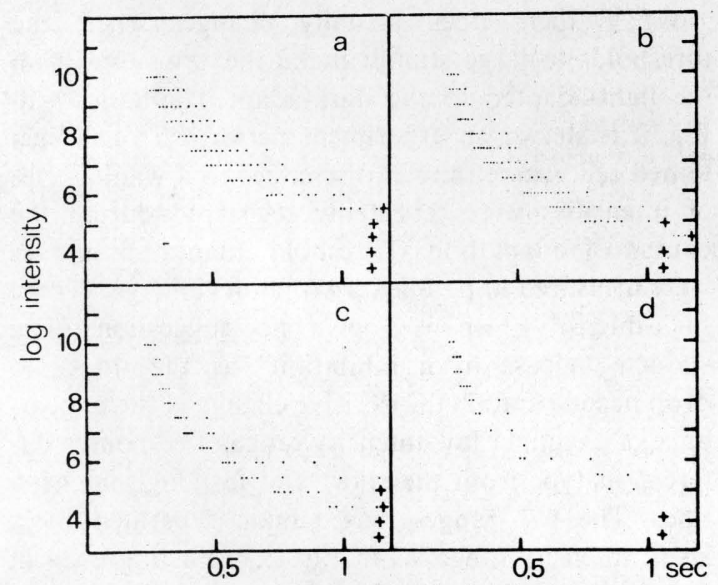

Fig. 2. How the time-course and impulse frequency of the discharges of a class 3 cell depend on stimulus size and intensity. Each dot is one spike, each row is one discharge to the stimulus intensity given on the ordinate. Abscissa: time, with stimulus onset at the left edge of each frame. Stimulus diameters were $0.11 \mathrm{~mm}$ (a), $0.3 \mathrm{~mm}$ (b), $0.8 \mathrm{~mm}$ (c) and $1.9 \mathrm{~mm}(\mathrm{~d})$. The + sign means that the discharge continued with spikes not included in the figure. first spike, here regarded as an excitatory latency $l_{e}$, is well described by an inverse log-function of the flux of quanta absorbed by the excitatory centre:

$$
l_{e}=\left(b \cdot \log \left(c I A_{e}\right)^{-1}=\left(b \cdot \log I_{r}\right)^{-1}\right.
$$

( $I=$ stimulus intensity, $A_{e}=$ the "effective" excitatory area stimulated, i.e. the integral of (area) (sensitivity of the excitatory mechanism) over the whole stimulating spot, $I_{r}=$ stimulus intensity relative to threshold, $b$ and $c=$ constants). This is seen from Fig. $4 \mathrm{a}$, where reciprocal latencies have been plotted as functions of $\log$ stimulus intensity for two different-sized stimuli: the result is two straight lines separated along the $\log I$ axis by the amount separating the corresponding thresholds. Figure $4 \mathrm{~b}$ shows that this linearity is preserved throughout adaptation, but the lines get steeper (the slope coefficient $b$ increases) with lightadaptation, and threshold latency decreases.

\section{DISCUSSION}

How can latencies determine responses and thresholds?

Considering equation (1) and the adaptational changes mentioned, the following hypothesis will account for both the discharge patterns of class 3 cells (Fig. 2) and the elevated phase of the threshold adaptation curves as measured with large stimuli. It is assumed that the inhibitory signal has a latency $l_{i}$ that can be described by a similar function as that of the excitatory latency $l_{e}$, but including a delay $d$ :

$$
\begin{aligned}
l_{i}=\left(b \cdot \log \left[c I A_{i}\right]\right)^{-1} & +d \\
& =\left(b \cdot \log \left[I_{r} \cdot A_{i} / A_{e}\right]\right)^{-1}+d
\end{aligned}
$$

( $A_{i}$ is the "effective" inhibitory area stimulated, defined by analogy with $A_{e}, d$ is the delay, which is assumed to be constant to a first approximation). The justification for this is that the inhibitory pathway differs from the excitatory pathway by an interposed amacrine cell (cf. Werblin and Copenhagen, 1974; 


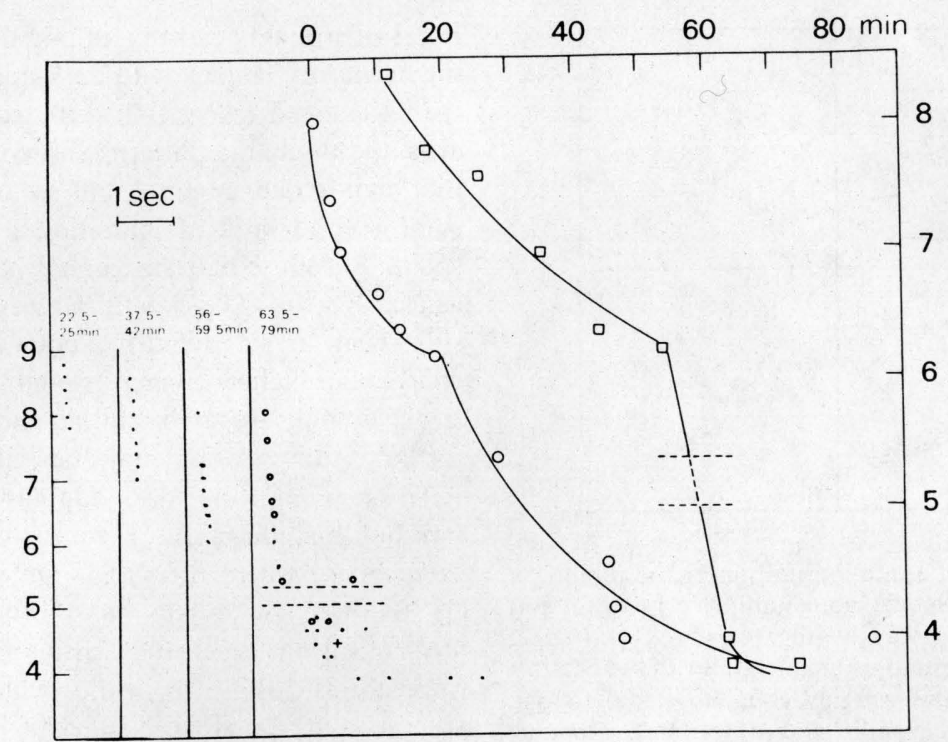

Fig. 3. Left: the discharge patterns of a sustained cell to a large $(1.9 \mathrm{~mm})$ stimulus registered at different times during dark-adaptation after a $3 \%$ bleach. The discharges are displayed as in Fig. 2 with log stimulus intensity on the ordinate and time on the abscissa; above each set is given the time interval (after bleach) during which it was registered. The broken lines mark the intensity range where no responses could be obtained with this stimulus. In the responses of 63.5-79 min, spikes recorded during the last $10 \mathrm{~min}$ are represented by small circles. Right: the corresponding threshold adaptation curves measured with a $1.9 \mathrm{~mm}$ spot (upper) and a $0.11 \mathrm{~mm}$ spot (lower). Temperature: $7.5-8.8^{\circ} \mathrm{C}$.

Miller and Dacheux, 1976). Concretely speaking, $l_{i}$ is represented by the same curve as $l_{e}$, but shifted by the amount $-\log \left(A_{i} / A_{e}\right)$ along the $\log$ intensity axis and by the amount $d$ along the time axis. The ratio $A_{i} / A_{e}$ is unknown for each spot and has to be calculated on the basis of some further assumptions, however, this is not critical here, as there will always be three main possibilities: $A_{i} / A_{e}<1$ (small spot mainly stimulating the centre), $A_{i} / A_{e} \simeq 1$ (moderately large spot stimulating centre and surround about equally) and $A_{i} / A_{e}>1$ (large spot strongly stimulating the surround). The possibilities are illustrated in Fig. 5 for small spots (a and b), an intermediate spot (c) and a large spot (d), using the same conventions as in Fig. 2, and setting $d=44 \mathrm{msec}$, which was the value giving the best fit with experimental discharge patterns. In (d), with the $l_{i}$ curve shifted relatively downwards, the $l_{i}$ and $l_{e}$ curves cross over. If no spikes could get through after the onset of inhibition, the $l_{i}$ and $l_{e}$, curves should constitute the time limits of the discharges at all test intensities. A comparison with Fig. 2 reveals a fair agreement, although especially at low intensities stray

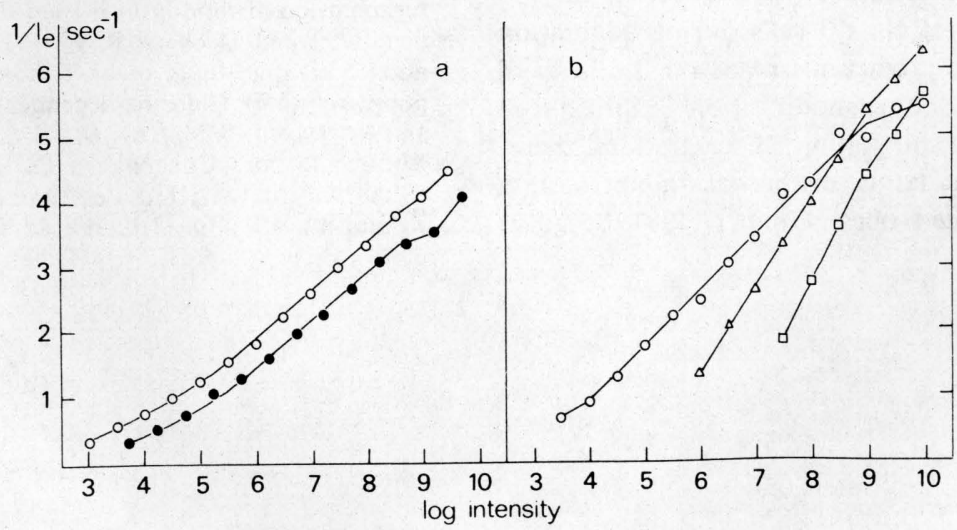

Fig. 4. (a) Reciprocal latency to the first spike $\left(I / l_{e}\right)$ as a function of log stimulus intensity for a dark-adapted cell. Stimulus spots $0.11 \mathrm{~mm}$ (filled symbols) and $0.8 \mathrm{~mm}$ (open symbols) dia. The lines drawn to fit the points are identical (except for the highest intensities), but shifted by the amount that separated the thresholds to the two spots. (b) How light-adaptation makes the straight lines giving reciprocal latency as a function of $\log$ stimulus intensity steeper. The responses to a $0.3 \mathrm{~mm}$ spot were registered in the dark-adapted state (circles) and after a $3 \%$ rhodopsin bleach: 4 min after (squares) and $20 \mathrm{~min}$ after (triangles). 


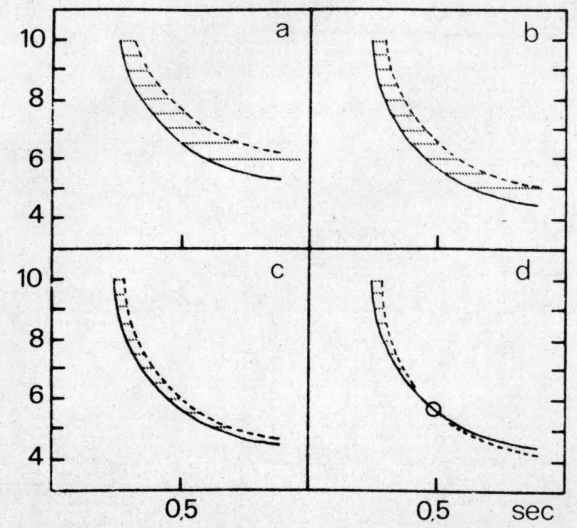

Fig. 5. The latencies of excitation (full lines) and inhibition (broken lines) as calculated from equations (1) and (2) and displayed in the same way as the responses in Fig. 2. (a) corresponds to a stimulus smaller than the excitatory centre, (b) to a stimulus roughly equal to it, (c) to one extending moderately beyond it and (d) to a large stimulus extending far into the surround.

spikes do occur after the expected onset of inhibition. These form the irregular low-intensity responses mentioned earlier, and will be termed post-inhibitory spikes.

Disregarding these for the moment, the condition for the threshold intensity to a large stimulus, obtained from equations (1) and (2), would be

$$
\begin{aligned}
l_{i}-l_{e}=\frac{1}{b} \cdot\left\{\left(\log I_{r}+\right.\right. & \left.\log \left[A_{i} / A_{e}\right]\right)^{-1} \\
& \left.-\left(\log I_{r}\right)^{-1}\right\}+d=0
\end{aligned}
$$

As $b$ decreases in a regular manner during dark-adaptation ( $c f$. Fig. $4 b$ ), this yields adaptation curves resembling the initial elevated phase of the experimental ones (equation (3) gives $I_{r}$ at each moment, i.e. threshold intensity in relation to the non-inhibited "small-spot" threshold intensity). Changes in the full discharge patterns of class 3 cells during adaptation also agree with the predictions made on the basis of latency-adaptation. Some modification is introduced by the fact that the summating RF centre (and thereby $A_{e}$ ) is somewhat larger in the dark-adapted state than in light-adapted ones (Donner, 1981, see also
Fig. 1a), probably causing an additional shortening of the excitatory latency with dark-adaptation.

In sustained (class 1-2) cells, contrary to class 3 cells, the discharge patterns show no undisturbed initial high-frequency burst, but seem to be affected by some second kind of inhibition from the very first spikes. Response patterns cannot be delimited in time as clearly as in class 3 cells. However, eqs (1) and (2) still seem to set definitive boundaries to the discharges at higher intensities and particularly when larger stimuli are used, and so threshold adaptation for large stimuli would still obey equation (3).

To sum up, the dark-adaptation of large-spot thresholds is interpreted as consisting of an initial elevated phase where thresholds are given by equation (3), followed by a late phase when the excitatory mechanism has reached a sensitivity sufficiently high to respond to those intensities where post-inhibitory responses are possible. Neither of these need involve any differences in the adaptation of the excitatory and the inhibitory pathways. During the first phase, adaptation is essentially determined by latency adaptation, seen as a change in the slope of the reciprocal-latencylines. The subsequent occurrence of post-inhibitory responses may be based simply on the statistical fluctuation of the excitatory and inhibitory inputs, which may be considerable at the lowest intensities (the absolute threshold was about 10 quanta absorbed by the excitatory mechanism within an integrating time of some $5 \mathrm{sec}$ ).

\section{REFERENCES}

Barlow H. B., Fitzhugh R. and Kuffler S. W. (1957) Change of organization in the receptive fields of the cat's retina during dark-adaptation. J. Physiol. 137, 338-354.

Donner K. (1981) Receptive fields of frog retinal ganglion cells: response formation and light-dark-adaptation. $J$. Physiol. 319. In press.

Donner K. O. and Reuter T. (1965) The dark-adaptation of single units in the frog's retina and its relation to the regeneration of rhodopsin. Vision Res. 5, 615-632.

Miller R. F. and Dacheux R. F. (1976) Synaptic organization and ionic basis of on and off channels in mudpuppy retina. II. Chlorine-dependent ganglion cell mechanisms. J. gen. Physiol. 67, 661-678.

Werblin F. S. and Copenhagen D. R. (1974) Control of retinal sensitivity - III: Lateral interactions at the inner plexiform layer. J. gen. Physiol. 63, 88-110. 